\title{
Liderazgo y clima organizacional de un hospital pediátrico cubano. Visión de enfermeras jefas y asistenciales*
}

Liderança e clima organizacional de um hospital pediátrico cubano. Visão de enfermeiras chefes e assistenciais

Leadership and organizational climate of a Cuban pediatric hospital. Vision of head and care nurses

\section{Annia Lourdes Iglesias Armenteros ${ }^{\mathrm{I}}$, Julia Maricela Torres Esperón ${ }^{\mathrm{II}}$, Yuliett Mora Pérez ${ }^{\mathrm{III}}$, Jose Rolando Sánchez Rodríguez ${ }^{\mathrm{IV}}$}

Resumen: Objetivo: describir experiencias vividas de enfermeras jefas y enfermeras asistenciales sobre las causas que pueden afectar el clima organizacional de los servicios pediátricos en un hospital cubano. Método: estudio cualitativo de tipo exploratorio realizado en los meses de abril-diciembre, año 2018 con 14 enfermeras jefas y 11 enfermeras asistenciales, pertenecientes al Hospital Pediátrico Universitario "Paquito González Cueto", Provincia de Cienfuegos, Cuba. Los datos se recogieron con la técnica de grupo focal, las narrativas fueron procesadas mediante análisis de contenido en base al referencial teórico de clima organizacional con la categoría central de liderazgo. La investigación fue evaluada por Comité Ético-Científico de hospital de referencia. Resultados: emergieron cuatro subcategorías del análisis de contenido con base en el referencial teórico. La de mayor peso fue "ideario de un directivo" seguida de "mejoramiento continuo" "escucha para la armonía relacional" y "sentido de pertenecía". Conclusiones: las principales causas que afectan el clima organizacional se relacionan con las características del líder y las posibilidades de superación de ellos y del resto del personal.

Descriptores: Liderazgo; Enfermería; Organización y administración; Administración hospitalaria; Ambiente de trabajo

\footnotetext{
${ }^{\text {I }}$ Enfermera. Doctora en Ciencias de la Salud. Profesor Auxiliar e investigador agregado. Universidad de Ciencias Médicas de Cienfuegos. Cienfuegos, Cuba. Email: anniaia@jagua.cfg.sld.cu Orcid: https://orcid.org/0000-0002-7031-0827

II Enfermera. Doctora en Ciencias de la salud. Profesor e investigador titular. Escuela Nacional de Salud Pública. Habana, Cuba. Email: mtorresesperon7@gmail.com Orcid: https://orcid.org/0000-0002-0702-7025

III Enfermera. Doctora en Enfermería. Profesor auxiliar e investigador agregado. Vicedirectora de Enfermería. Hospital Provincial Gustavo Aldereguía Lima. Cienfuego, Cuba. Email: yuliett.mora@gal.sld.cu Orcid: https://orcid.org/0000-0003-3393-8081

IV Enfermero. Doctor en Enfermería. Profesor Asistente de Enfermería. Universidad Arturo Prat. Sede Victoria. Chile. Email: josersan@unap.cl Orcid: https://orcid.org/0000-0001-7347-028X

* Trabajo extraído de la tesis doctoral: "Intervención para la mejora del clima organizacional de enfermería. Hospital Pediátrico Universitario "Paquito González Cueto", Cienfuegos 2012-2018, presentada en la Escuela Nacional de Salud Pública. Universidad de Ciencia Médicas de La Habana, Cuba.
} 
Resumo: Objetivo: descrever as experiências vividas por enfermeiras chefes e enfermeiras assistenciais sobre as causas que podem afetar o clima organizacional dos serviços pediátricos em um hospital cubano. Método: estudo qualitativo do tipo exploratório, realizado nos meses de abril a dezembro de 2018, com 14 enfermeiras chefes e 11 enfermeiras assistenciais, pertencentes ao Hospital Pediátrico Universitário "Paquito González Cueto", Província de Cienfuegos, Cuba. Os dados foram coletados por meio da técnica de grupo focal, as narrativas foram processadas por meio da análise de conteúdo, com base no referencial teórico de Clima Organizacional, com a categoria central de liderança. A investigação foi aprovada pelo Comitê Ético-Científico do hospital de referência. Resultados: emergiram quatro subcategorias de análises de conteúdo com base no referencial teórico. A de maior peso foi "Ideología do gerente", seguida por "Melhoramento contínuo", "Escuta para a harmonia relacional" e "sentido de pertencimento". Conclusões: as principais causas que afetam o clima organizacional se relacionam com as características do líder e as possibilidades de superação delas e do restante da equipe.

Descritores: Liderança; Enfermeira; Organização e administração; Administração hospitalar; Ambiente de trabalho

Abstract: Objective: Describe experiences of nurses heads and clinical nurses on the causes that may affect the organizational climate of pediatric services in a Cuban hospital. Method: Qualitative and exploratory study done in the months of April - December, 2018 with 14 heads nurses and 11 healthcare nurses, belonging to the Pediatric University Hospital "Paquito Gonzales Cueto", Province of Cienfuegos, Cuba. The data were collected with the focus group technique; the narratives were processed through content analysis based on the theoretical framework of organizational climate with the central category of leadership. The research was evaluated by the Ethical-Scientific Committee of the reference hospital. Results: Four subcategories of content analysis emerged based on the theoretical framework. The most important was "ideology of a manager "followed by "continuous improvement" "listening for relational harmony" and "sense of belonging".

Conclusions: the main causes as affecting organizational climate are related to the characteristics of the leader and the possibilities of overcoming them and the rest of the staff.

Descriptors: Leadership; Nursing; Organization and an administration; Hospital administration; work environment

\section{Introducción}

El Sistema Nacional de Salud en Cuba se plantea como desafío el perfeccionamiento de la gestión de sus directivos en las instituciones de salud para lo cual considera objetivos esenciales como fomentar la creatividad e innovación de sus líderes corporativos mediante la formación permanente, en busca de la eficiencia, además de profundizar en las buenas prácticas internacionales relacionadas con la 
conducción y liderazgo de los recursos humanos y la una constante evaluación del proceso directivo. ${ }^{1}$

Los hospitales actúan en contextos complejos donde predominan las relaciones humanas, la incertidumbre y turbulencia de los procesos internos, como consecuencia de factores como el acelerado cambio tecnológico, las crisis económicas, el inusitado aumento de demandas sociales, la permeabilidad del estado conseguida por la sociedad y la gestión de recurso económico. Por lo que estudiar la dinámica de la gerencia de directivos de enfermería de hospitales, es fundamental para anticiparse estratégicamente al mejoramiento del entorno laboral, en una realidad donde la complejidad, el escepticismo y el conflicto sean retos permanentes de intervención, ${ }^{2}$ para fortalecer el clima organizacional, como elementos a considerar en los métodos organizativos de gestión, cambio e innovación, donde los funcionarios estén satisfecho con la labor que cumplen, por lo que estudiar este constructo brindaría retroalimentación en todos los procesos que influyen en el comportamiento de la organización y conducta de los individuos. ${ }^{3}$

En este sentido, la tendencia actual sobre gestión y liderazgo de enfermería en los servicios señala que ésta se considera un factor esencial e inherente a todas las teorías organizacionales. Para enfermería es una competencia fundamental en la estructura organizativa de instituciones de salud, debido a necesidad de contar con instituciones eficientes y competitivas, con recurso humano preparado en habilidades de gestión y conducción del recurso humano, que fomente las relaciones humanas centradas en la cultura y clima organizacional; al tiempo que genere procesos de análisis y posibilidad de cambio en las organizaciones, ${ }^{4}$ para así lograr una mejor calidad de atención al paciente, impacto en el ambiente de trabajo y bienestar del personal. ${ }^{5}$ 
Liderazgo \& clima organizacional de un hospital pediátrico cubano. Visión de ...| 4

Tradicionalmente, los profesionales de enfermería han emergido como buenos gerentes del equipo de salud, debido a su formación y desarrollo profesional, con amplia visión del "ser humano", del "cuidado" y de la "salud", más allá de lo visible, que articula conocimientos y acciones necesarias para ofrecer asistencia creativa y de calidad. Poseen capacidad de coordinar equipos y desarrollar estrategias para el cumplimiento de metas y objetivos propuestos por los sistemas de salud. ${ }^{6,7}$ No obstante, en muchos contextos se reconoce la invisibilidad para que los profesionales de enfermería puedan insertarse como líderes reconocidos al interior de los equipos de salud y que se respete su autonomía decisional. ${ }^{6}$

Es así como los profesionales de enfermería a través de la historia han incursionado en la gestión de hospitales y sus dependencias, al responder a paradigmas nuevos de gestión, con características de complejidad, pero siempre integrados a la gerencia pública, con estrategias de conducción del talento humano; en la cual las variables de reclutamiento, perfeccionamiento, conducción y seguimiento del equipo de trabajo, ha sido preocupación del sector sanitario cubano. ${ }^{2}$

La investigación se realizó en el Hospital Pediátrico Universitario "Paquito González Cueto" de Cienfuegos, único de la provincia, donde se presta atención médica de alta complejidad, para la población de ocho municipios. Posee 140 camas para la hospitalización distribuidas en 14 salas en las que laboran 148 enfermeras/os que equivalen a una proporción de una enfermera por 13 pacientes. Además, posee cuatro departamentos (urgencia, central de esterilización, consulta externa y unidad quirúrgica).

Entre el año 2012 y 2014 confluyeron varios aspectos en esta institución relacionados con el clima organizacional, visibilizados por los resultados de las encuestas anuales que miden la satisfacción del personal de enfermería, donde se obtuvo un $85 \%$ de insatisfacción, los resultados de los informes de la vice-dirección 


\section{5 | Armenteros LI, Esperón JMT, Pérez YM, Rodríguez JRS}

de Enfermería, reflejaban 71 renuncias correspondiente al equipo de enfermería del centro. Entre las causas relacionadas con el clima se mencionaban la excesiva carga de trabajo, la falta de motivación por este, se suman, además otros factores como el trato que reciben de los directivos de enfermería y la falta de reconocimientos y retribución a sus esfuerzos.

Todo ello generó un proyecto de investigación basado en una intervención para la mejora del clima organizacional en enfermería, ${ }^{8}$ los resultados de la fase cuantitativa (pre-experimento) apuntaron a la no mejoría del liderazgo después de la intervención. Fue por ello que se requirió indagar cualitativamente las causales que originaron que esta dimensión obtuviera resultados contraproducentes de acuerdo a las acciones realizadas. De ahí que el objetivo de este artículo fue; describir experiencias vividas de enfermeras jefas y enfermeras asistenciales, sobre las causas que pueden afectar el clima organizacional de los servicios pediátricos en un hospital cubano.

\section{Método}

Como parte de los resultados del estudio primario, de diseño mixto explicativo secuencial; (intervención para la mejora del clima organizacional en enfermería); emerge de la fase cuantitativa la necesidad de buscar explicaciones del porqué la dimensión de liderazgo no mejoró después de la intervención, que dió lugar a la fase cualitativa con diseño de tipo exploratorio, el que condujo a la descripción e interpretación de significados, perspectiva, visiones y percepciones relativos a las experiencias vividas por enfermeras con cargos de jefatura y enfermeras asistenciales, respecto a causales que afectan el clima organizacional de servicios pediátricos hospitalarios. Las unidades de análisis fueron profesionales de enfermería con cargos

directivos y enfermeras de servicios clínicos sin cargos gerenciales, todas 
Liderazgo \& clima organizacional de un hospital pediátrico cubano. Visión de ...| 6 pertenecientes al Hospital Pediátrico Universitario "Paquito González Cueto" de la Provincia de Cienfuegos, Cuba, durante los meses de abril-agosto, año 2018.

Para la selección de los participantes se tuvo presente, el universo de enfermeras con cargos directivos o sea que fueran jefas de salas, todas con más de 5 años como en el cargo. En el caso de las enfermeras asistenciales el criterio de selección fue que tuvieran más de cinco años laborando en el hospital, de las cuales se logró la aceptación de una de cada servicio, por lo que participaron 11 enfermeras.

Previamente a la indagación, se profundizó en la fase de vagabundeo para organizar los mapas temporales, con el objetivo de volver a vincularse con población de estudio, se continuó con aviso a participantes sobre propósito de investigación y se confirmó el anonimato, así como ratificaciones de confidencialidad de la información. Se resaltó, que jamás existiría represalias administrativas por información obtenida y con firma del consentimiento informado; previo aviso con cada grupo de profesionales de enfermería. Se realizó una sesión de grupo focal de una hora con cada grupo de participantes por separado (enfermeras con cargo directivo y enfermeras asistenciales); ambos con la misma pregunta guía a partir de la interrogante detonadora: ¿cuáles características ustedes consideran que debe tener la dirección del servicio para lograr buenos resultados de trabajo? Dicha pregunta estuvo relacionada con los resultados discordantes obtenidos en la fase cuantitativa de la investigación primaria, ${ }^{8}$ donde la dimensión liderazgo fue la única que no mejoró después de la intervención.

Dado el interés del estudio para identificar las causas de las respuestas negativas en la dimensión liderazgo las cuales ocasionaron alteraciones en el funcionamiento del clima organizacional, se decide como técnica de recogida de datos el grupo focal. La decisión obedece a lo planteado en la literatura científica, 
donde se plantea que la técnica es particularmente útil para explorar los conocimientos y experiencias de las personas en un ambiente de interacción, que permite examinar lo que la persona piensa, cómo piensa y por qué piensa de esa manera. ${ }^{9}$

Para realizar la técnica de grupo focal, se consideraron las fases de desarrollo de los métodos grupales: planteamiento de objetivos, selección de participantes, preparación, organización y desarrollo de las discusiones, análisis de información, así como selección del lugar de realización, el cual poseía las condiciones estructurales de espacio, ventilación y buena iluminación. Se dispusieron los asientos en forma de herradura para permitir visibilidad e intercambio entre participantes y la moderadora. Se contó con medios de grabación de la técnica, aspecto necesario para su posterior análisis de contenido.

Cada grupo focal se materializo individualmente, en sala multiuso del hospital, espacio alejado de dinámica y contexto asistencial de enfermería, a fin de evitar temores y aprehensiones durante los discursos. Su realización estuvo a cargo de una investigadora alterna, dos investigadoras participaron como observadoras y dos como auxiliares de la investigación.

Las dos sesiones de grupo focal transcurrieron en varios momentos, en el primero; la moderadora explicó los objetivos, la importancia de los diálogos, el valor de cada opinión y dinámica de trabajo que se utilizaría. Durante la fase central, la moderadora dirigió la conversación hacia la temática esencial a partir de la introducción de la pregunta investigativa. El debate transcurrió de manera armónica, en base al respecto y con la contribución de las participantes.

Las narrativas fueron grabadas, transcritas y agrupadas en un cuerpo textual. Con el fin de preservar el anonimato de participantes, se identificaron con letra [J] las jefas de sala y letra [E] las enfermeras asistenciales y fueron enumeradas de acuerdo 
Liderazgo \& clima organizacional de un hospital pediátrico cubano. Visión de ...| 8 con el orden de la palabra. Para el procesamiento de información obtenida, se utilizó la técnica de análisis de contenido de Bardin ${ }^{10-11}$ con el empleo de sus tres fases correspondiente: 1) pre-análisis, 2) exploración del material y 3) tratamiento de resultados, inferencia e interpretación.

En el pre-análisis, se organizó el cuerpo textual y se realizó lectura fluctuante con la finalidad de apropiarse de las ideas iníciales. Se decidió utilizar el método colorimétrico para seleccionar las unidades de análisis. En la segunda fase de exploración del material fue efectuada la lectura exhaustiva del cuerpo textual, momento en que los datos brutos son transformados, organizados y reunidos en unidades, que permiten la descripción de las características pertinentes del contenido, se marcó en diferentes colores las unidades análisis, se buscaron los núcleos de sentido que dieron lugar a las categorías.

En la tercera fase de tratamiento de los resultados, inferencia e interpretación se realizó el análisis categorial en base a las categorías del referencial teórico relacionada con la dimensión liderazgo del clima organizacional. Se aplicó la frecuencia ponderada que permitió identificar el peso de la frecuencia de cada categoría.

La investigación fue evaluada y aprobada con el acuerdo $\mathrm{N}^{\circ} 25-18$, del 23 de julio de 2018; por Consejo Científico y Comité de Ética, del hospital de referencia, posteriormente se desarrolló colecta de datos, en cumplimiento con principios básicos de la Declaración de Helsinki de la Asociación Médica Mundial, en su versión de la 59å Asamblea General, Seúl, Corea, octubre 2008. Además se cumplió en cada fase de la investigación, con los requisitos éticos de Ezequiel Emanuel. ${ }^{12}$ Se cautelaron principios bioéticos como, respeto a la autonomía: en derecho del participante a expresar opiniones, decisiones y participación o no en investigación, además de nulas repercusiones personales, al contribuir al estudio; de beneficencia: al no existir 
9 | Armenteros LI, Esperón JMT, Pérez YM, Rodríguez JRS

acciones, condiciones, daños, riesgos, malestar o dolor para los participantes, respetando sus derechos; de no maleficencia: por no ocasionar ningún tipo de peligro físico o emocional y el principio de justicia: al tratar a todos los participantes de manera similar y con oportunidad de contribución. ${ }^{12}$

\section{Resultados}

El instrumento utilizado en la fase cuantitativa del estudio mixto secuencial explicativo, fue el "inventario de clima organizacional”, el cual mide cuatro dimensiones básicas: liderazgo, motivación, reciprocidad y participación, las que a su vez se subdividen en cuatro variables cada una. En el caso de la dimensión liderazgo, las variables son responsabilidad, comunicación, estímulo a la excelencia y dirección. Según datos obtenidos en la evaluación de esta fase, la dimensión liderazgo fue la de resultados menos favorables de manera general en ambas unidades de análisis. ${ }^{8} \mathrm{De}$ ahí la necesidad del presente estudio de diseño exploratorio (fase cualitativa) en la que se asumió como categoría central “el liderazgo”, de ahí que en el análisis de las subcategorías emergentes se pudiera realizar una concatenación con las variables de la dimensión liderazgo. A modo de resumen se organizan la categoría central y las subcategorías en la siguiente Figura 1.

Figura 1- Mapa de fragmentos del corpus de datos. 


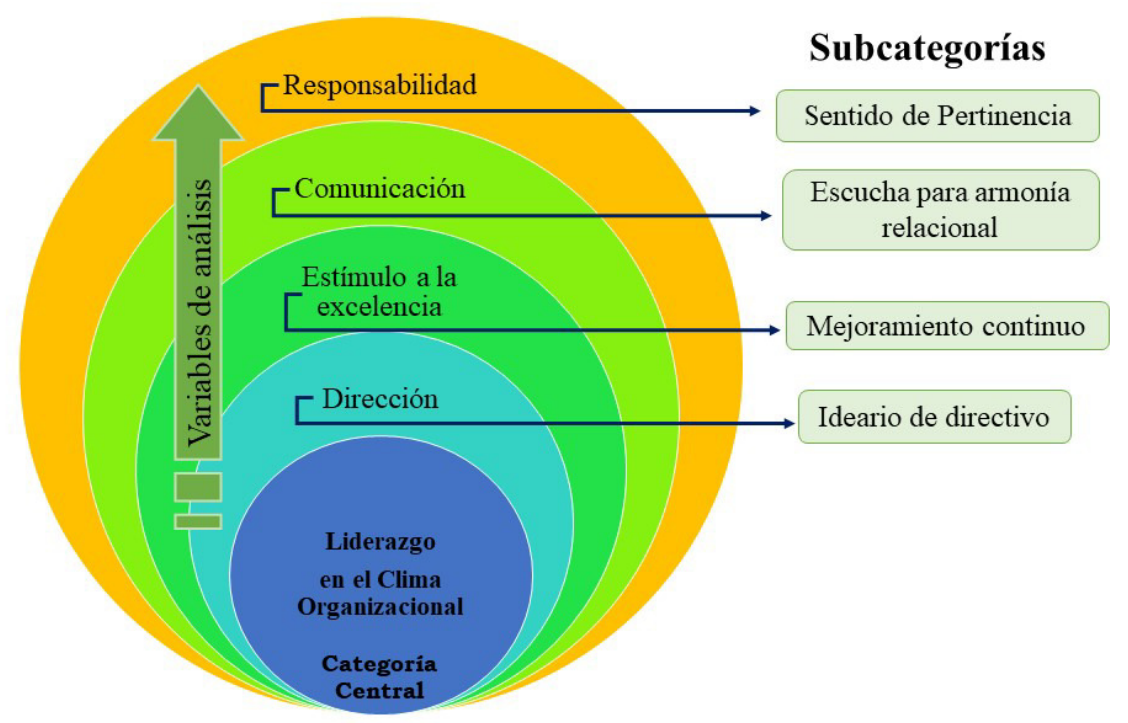

Fuente: base de datos de investigación. Elaboración propia.

En el procesamiento de las narrativas de los dos grupos focales, emergieron cuatro subcategorías del análisis de contenido con base en el referencial teórico. La de mayor peso fue "ideario de un directivo" seguida de "mejoramiento continuo" "escucha para la armonía relacional” y "sentido de pertenecía” Cada una de ellas se describen a seguir.

\section{Ideario de directivo}

Esta subcategoría se relaciona con la variable dirección de la dimensión liderazgo con la que se midió el Clima Organizacional en el estudio cuantitativo ${ }^{8}$ y los resultados se enfocan básicamente en las características que según las participantes debe distinguir a un directivo para ser más efectivo en su gestión y conducción del talento humano, los discursos que la sustentan se presentan a seguir.

[...] trabajar en unión, en colectivo, dirigir a la misma vez, que todo el mundo este concatenado, que todos se lleven bien (J2), [...] llevarse bien con todos los trabajadores que están a su lado (J7), [...] tener una buena relación con todo el personal (J10), [...] ser 
ejemplo [...] lo primero que debe ser el jefe es ser ejemplo, la persona que se subordina sigue siempre el ejemplo de él(J2), [...] que el jefe sea ejemplo (J3), ...] para ser un jefe hay que ser ejemplo, para que te vean como ejemplo (J7), [...] debe ser líder y ejemplo ante el resto del colectivo (J10), ...] tiene que saber escuchar y ser escuchado (J7), [...] saber escuchar sus problemas (J10) [...] cualquier dirigente del hospital lo primero que tiene que hacer es tratar a todos los trabajadores con respeto (E5), [...] que se respeten tantos unos como otros (J2), [...] cuando hay respeto entre dirigente y el trabajador el trabajo fluye mejor. (E5)

\section{Mejoramiento continuo}

En este caso la subcategoría se relaciona con la variable estímulo a la excelencia de la dimensión liderazgo y apunta a la necesidad de superación y capacitación permanente, tanto de enfermeras directivas como asistenciales para el logro de los objetivos de la institución pediátrica, pero sobre todo para el mejoramiento de la calidad de la atención.

[...] para ser un buen dirigente, se debe estar muy bien preparados tanto administrativo como científico, como en lo técnico (J5) [...] el alto nivel científico para dirigir a los demás subordinados (E7), [...] hacer énfasis con el personal nuevo, con los estudiantes que se están incorporando (J2) [...] debe prepararse el personal nuevo ya para el relevo, sobre todo en los servicios especializados (E6), [...] los que en estos momentos estamos dirigiendo los servicios nos corresponde trabajar en esa parte [...] en la preparación porque eso es el futuro de nosotros (J2), es importante la superación sobre todo ser Profesional, que den clases práctica o capacitar en otros servicios (E3), [...] la investigación es importante (J9), hay que elevar el nivel científico porque elevando el nivel científico se eleva la calidad en el trabajo (E6) [...] la persona que dirige siempre tiene que estar arriba de las demás personas, en la docencia [...] saber 
Liderazgo \& clima organizacional de un hospital pediátrico cubano. Visión de ...| 12

siempre, estar estudiando para que pueda dirigir (J12) [...] se requiere que estén bien capacitados para trabajar con niños.(E8)

\section{Escucha para armonía relacional}

Esta se corresponde con la variable comunicación, en ella se le da un valor especial a la escucha sintonizada y resonante por parte de los directivos, como habilidad fundamental para el trabajo en equipo.

[...] la unidad del servicio partiendo de...quien lo dirige hay que saber siempre escuchar, tanto al personal que no es el jefe del servicio y a los demás porque así uno trabaja más unido [...] y las cosas marchan mejor y si se logran buenos resultados porque todo el mundo se ve como un equipo íntegro y todo marcha mejor (J9), [...] se ha perdido un poco de disciplina, un poco de la parte de educación formal (J2), [...] es importante que el personal de enfermería mantenga una relación, que todos los trabajadores del sector de la salud, que todo el mundo aprenda a tratar de llevarse bien y a respetarse entre sí. (E3)

\section{Sentido de pertenencia}

Esta última subcategoría se relaciona con la variable responsabilidad, en ella los discursos se encaminan a las circunstancias de satisfacción de una persona, al formar parte de un grupo, una comunidad u otro tipo de conjunto, donde se valoriza la institución como propia y con ello progresar en el trabajo colectivo.

[...] uno tiene que tener sentido de pertenencia desde que entra por la puerta del hospital, de todo lo que está ahí dentro del servicio de cada cual y dentro de la institución, cuidar todo. (J12) [...] pensar que este es mi hospital y estos son mis niños. (E8) [...] yo amo este pediátrico trabajo aquí desde que me gradué. (J9)

\section{Discusión}

Los investigadores reconocen en los participantes, que sus discursos están matizados por intereses, demandas y aspiraciones, para que se equilibre en su 
institución y/o servicios un buen clima laboral, el cual se proyecte hacia el respeto, a las buenas relaciones humanas, al modelo de líder que los dirige y hacia el logro de objetivos generales de la institución. Por ello la importancia de pesquisar el comportamiento de este constructo a fin de continuar procesos capacitantes para la mejora continua del clima organizacional que influyan en el ambiente de trabajo y con ello evitar situaciones de conflicto o malestar de los funcionarios que repercutan en el rendimiento en sus desempeños de cuidados.

La dirección, es vista como método que deben aplicar los directivos para la conducción del recurso humano, donde se evidencie en un gestor, el modelo a seguir, condicionados por el apoyo y unión colectiva para suplir necesidades laborales e involucrarse en el dominio sobre las situaciones administrativas y asistenciales del servicio que dirige un jefe, donde los profesionales sientan que sus esfuerzos por mantener el funcionamiento integral de su servicios se distinga, al reflejarse un trabajo en equipo que se diferencia por sus buenas relaciones humanas, como se destaca en el estudio ${ }^{13}$ donde concluye que la relación supervisor con su equipo de trabajo son determinantes importantes del vínculo afectivo de los profesionales de enfermería con sus organizaciones de salud.

Dentro de la dirección el liderazgo ocupa un lugar determinante, así lo se refleja en los estudios realizados, ${ }^{14-16}$ donde se destaca que los profesionales de enfermería de diferentes entornos culturales, geográficos y ocupacionales tenían diferentes interpretaciones sobre el clima y cultura organizacional y construcciones subyacentes del estilo de liderazgo. Lo central para el trabajo en equipo interdisciplinario efectivo, es la forma y estilos de dirección para conducir los recursos humanos, al reconocer y construir relaciones laborales sobre las diferencias 
Liderazgo \& clima organizacional de un hospital pediátrico cubano. Visión de ...| 14 únicas en los estilos de comportamientos de los miembros del equipo, así como el propio método directivo del dirigente.

En el ideario directivo; no se trata de personalidad; se trata de comportamientos humanos, para valorar y comprender las diferencias de estilos de dirección que ayuden a mejorar la autoestima y la satisfacción entre los equipos de enfermería e identificar y utilizar habilidades inherentes de las personas. Para los investigadores es esencial esta subcategoría, debido a que la acción de dirección está condicionada, a perspectiva gerencial del jefe con su equipo de trabajo, objetivando, bienestar del personal, consideración del ejercicio y compromiso laboral, traducidos en responsabilidad, empatía, habilidad para toma de decisiones y una comunicación y la administración de forma efectiva y eficaz. Resultados que hacen analogía con otros estudios, ${ }^{13,17-18}$ donde resaltan que, para un buen trabajo en equipo interdisciplinario, se requiere énfasis en la cooperación, colaboración y comunicación como predictores del compromiso laboral y el apoyo social del supervisor.

Desde esta perspectiva, el análisis realizado por otros autores ${ }^{17,19}$ sobre este tema, encontraron que existen significativas evidencias de que la confianza en un líder está fuertemente relacionada con actitudes en el trabajo, en segundo lugar, y en menor grado, con conductas ciudadanas $\mathrm{y}$, por último, con resultados organizacionales. Por ejemplo, investigaciones recientes han encontrado un impacto significativo en: compromiso organizacional, ${ }^{16}$ sentido de empoderamiento, ${ }^{15}$ percepción de efectividad del líder ${ }^{14} \mathrm{y}$ satisfacción con el trabajo. ${ }^{7}$

En la subcategoría mejoramiento continuo, los participantes la perciben como un indicador relevante en la motivación para el trabajo. De los análisis emerge la necesidad de superación constante tanto de enfermeras directivas como de enfermeras asistenciales y el valor que le confieren para el desarrollo personal e 
institucional; sumado a varios factores que median el clima organizacional; algunos se relacionan con preparación científico técnica del dirigente, la personalidad individual, los adiestramientos en servicios especializados y la formación continua, todos configuran una parrilla de diversas variables que favorecen el entorno laboral, las relaciones entre compañeros y el liderazgo. Resultados que hacen semejanzas con el estudio, ${ }^{13}$ donde concluye que la capacitación y formación de los supervisores en estilos de liderazgo, entrenamientos gerenciales y manejos de recursos laborales en el trabajo, permitirían aumentar los niveles de compromiso afectivo de los recursos humanos en las unidades o servicios de enfermería.

Según un artículo $^{20}$ sobre la evolución y perfiles de la enfermera quirúrgica y gestora en el que, entrevista a una profesional de más de 40 años de experiencia, ésta declara que

el mando intermedio o mando superior, primeramente, debes tener conocimientos. No tanto a nivel de dirección de enfermería, supervisora o adjunta, debes ser experta en las unidades que vas a gestionar. Porque si tienes una amplia experiencia en estas unidades sabes sacar el mayor provecho posible. Segundo, también tienes que tener conocimientos de gestión. ${ }^{20: 5}$

La subcategoría escucha para armonía relacional, visiblemente relacionado con la comunicación, se consideró esencial por las participantes del estudio para la garantía del clima organizacional y con ello el buen funcionamiento del equipo, aspecto que incluso para enfermería reviste gran valor, dado que es reconocido como uno de los atributos del cuidado humanizado, holístico y parsimonioso. Al decir de una enfermera académica chilena,

[...] el atributo de la comunicación permite comprender la interacción en el acto de comunicar y cuidar; desencadenaría el beneficio mutuo entre personal de salud y el sujeto del cuidado, pero, actualmente se evidencia acotado la realización del acto del cuidado, dando en la teoría y la práctica realce a la atención humanizada. ${ }^{21: 29}$ 
Liderazgo \& clima organizacional de un hospital pediátrico cubano. Visión de ...| 16

En esta subcategoría se hace hincapié en la capacidad que debe tener en jefe de enfermería para escuchar atentamente a su equipo de trabajo, en busca de comprender emociones, sentimientos e inconvenientes que pudieran perturbar el clima organizacional del servicio de enfermería y con ello las relaciones internas de sus miembros. El poder de escucha es un interés verdadero, autentico y de esta habilidad se proyecta el respeto mutuo, de lo contrario aparece la frustración del equipo de trabajo. Resultados como los acá descritos se relacionan con otros estudios, ${ }^{17}$ donde concluye que los líderes de enfermería pueden beneficiarse de la conciencia de las diferencias en las preferencias de estilo de comportamiento para mejorar la comunicación y la efectividad del equipo, así como mejorar la satisfacción entre los miembros del colectivo de trabajo.

Para los investigadores, resulta importante destacar que, en los equipos de trabajo de enfermería la comunicación es el único vehículo para relacionarse y establecer nexos estrechos por medio de los lenguajes, tanto verbal como no verbal, implícitos en cada cultura en particular y si a esto se le agrega el poder de escucha; serían procesos ineludibles y de gran importancia en las relaciones humanas, considerada de gran utilidad tanto a nivel personal como profesional. En un estudio ${ }^{22}$ sobre la comunicación en los gestores de enfermería: un papel fundamental; concluye que existe un déficit de comunicación o fallo de esta, entre el equipo y los directivos, por lo que se hace necesarios la importancia de la comunicación en la gestión y la imperiosa necesidad formativa en la competencia comunicativa para la gestión eficiente de servicios de enfermería.

Finalmente, la subcategoría, sentido de pertenencia, vinculada a la variable responsabilidad, denotan el compromiso que se precisa por parte de los participantes con su institución y permite analizar la necesidad de acudir a las personas 
comprometidas con el hospital como fortaleza para generar cambios, siempre que se den las condiciones para sentirse aceptado como miembro de un equipo, donde medie el respeto y armonía del grupo.

La pertinencia en el medio laboral de enfermería guarda relación con las tareas asignadas a los funcionarios de los equipos, la cual pudiera tener resultados favorables en la medida que las condiciones del clima laboral, ambientes expansivos de relaciones humanas y la comunicación, sean efectivas dentro del espacio de trabajo. Es acá donde el estilo de dirección del enfermero jefe puede favorecer el desempeño óptimo de la tarea, por parte del equipo de trabajo, el cual se constituye y tiene su sentido por y en la tarea, incluso quien recibe la acción encomendada, puede percibir ciertos grados de satisfacción.

Por lo que es indispensable proporcionar a los equipos de trabajo suficiente información, así como orientación técnica para la realización de diferentes actividades a desarrollar, además de persuadirlos, del porque es necesario su cumplimiento, de ningún modo imponer las actividades y orientaciones. En este sentido, es necesario dar oportunidad de participación en la toma de decisiones, en la solución de conflictos, en la responsabilidad ante el cambio, para que los equipos de enfermería se sientan identificados y comprometidos con su institución; a su vez más motivados y reconocidos. De ahí la importancia que muchos conocedores del tema le dan a la comunicación, para obtener grados de responsabilidad y más que todo, sentido de pertinencia de su institución.

Estos resultados se corresponden con el estudio sobre Clima Organizacional: Percepción por enfermeras del Nivel Primario de Atención, ${ }^{23}$ donde recomienda que es vital que a los equipo de salud y en especial los de enfermería, se les estimule haciéndolos participes de las decisiones de su servicio e institución, al 
Liderazgo \& clima organizacional de un hospital pediátrico cubano. Visión de ...| 18 comprometerlos con las políticas institucionales respectivas; y que no sólo se motive al funcionario con incentivos económico, sino con reconocimientos de tipo personal, que se sientan respaldados por la organización, motivados, de esta forma serán más responsables y podrán desplegar toda su creatividad en post de alcanzar las metas y objetivos de la institución.

Esta subcategoría, sentido de pertenecía, también permite aludir al concepto "sentido de comunidad" el que consiste en la percepción de las semejanzas con los otros, identidad social compartida, interdependencia, influencia, cohesión y unión de un grupo, relación de afecto, haciendo por los otros lo que ellos harían por usted, compartiendo sentimientos y responsabilidades. ${ }^{24}$

Los resultados de esta investigación hacen analogías con un estudio sobre sentido de comunidad de un equipo multidisciplinario de salud refieren que los participantes identifican el sentido de pertenecer al equipo principalmente por la capacidad de los profesionales de trabajar conjuntamente, respetar el lugar del otro, y principalmente, por percibir su importancia para el grupo y sentirse miembros de él. De la misma forma, el reconocimiento de que el trabajo del sector funciona cuando el profesional es parte del equipo y de los resultados alcanzados mostraron un elemento significativo para mantener a las personas integradas en un mismo propósito. ${ }^{25}$

Otros estudios sin embargo encuentran efectos contrarios, cuando registran que el sentido de pertenencia hacia la profesión de enfermería es incipiente. Esta motivación no alcanza a desarrollarse porque las relaciones y la convivencia no son del todo buenas, principalmente porque existen problemas no resueltos entre las enfermeras asistenciales. Otros factores que dificultan el desarrollo del sentido de pertenencia son la falta o pérdida de la integración de los miembros y la insatisfacción de algunos participantes por el trabajo que desempeñan en el servicio. ${ }^{26}$ 
Ambos análisis confirman la importancia de un buen clima organizacional para fortalecer el sentido de pertenencia, en el que liderazgo y trabajo en equipo son piezas fundamentales para ello.

\section{Limitaciones del estudio}

En Cuba, en el área de enfermería existen escasos estudios publicados con relación al tema. Se reconoce inclusión en esta investigación de artículos originales, pudiendo existir sesgo de publicación, por información científica en bases de datos de congresos y simposios que no pudieron ser incluidas al no tener acceso.

\section{Conclusiones}

Las principales causas que afectan el clima organizacional están relacionadas en la dimensión liderazgo se relacionan básicamente con las características de los líderes y las posibilidades de superación de ellos y del resto del personal, las que redundan en mejores relaciones laborales y son insumos básicos que sustentan el clima de la institución de enfermería. La comunicación y la responsabilidad subyacen entre otros umbrales necesarios para alcanzar, mejores relacionales de trabajo, y con ello perfeccionar las dinámicas, afectivas, comunicativas y de pertenencia de los equipos de trabajos de enfermería.

Los resultados de este estudio aportan una mirada al tema de la gestión hospitalaria, en particular en el área de enfermería, en tanto visibilizan causas que afectan el clima organizacional con especificidad en la dimensión liderazgo, como uno de los aspectos necesarios para abordar en la gerencia de los servicios de enfermería. No obstante, los hallazgos revelados en el estudio no consigan generalizarse, situaciones como éstas pueden ocurrir en cualquier institución de 
Liderazgo \& clima organizacional de un hospital pediátrico cubano. Visión de ... 20

salud como parte de las dinámicas de relación de los equipos, por lo que las categorías encontradas, pudieran ser transferibles a contextos similares.

\section{Referencias}

1. Hernández Nariño A, Delgado-Landa A, Marqués-León M, Nogueira-Rivera D, Medina-León A, Negrín-Sosa E. Generalización de la gestión por procesos como plataforma de trabajo de apoyo a la mejora de organizaciones de salud. Rev Gerenc Políticas Salud; 2017;15(31). doi: https://doi.org/10.11144/Javeriana.rgyps15-31.ggpp

2. Perea Vásquez LE, Rojas Torres IL. Modelos de gestión en instituciones hospitalarias. Rev Gerenc Políticas Salud [Internet]. 2019 [citado 2020 feb 05];18(36). Disponible en: https://revistas.javeriana.edu.co/files-articulos/RGPS/18-36\%20(2019-I)/54559086013/

3. Iglesias Armenteros AL, Torres Esperón JM, Solís FR. Un acercamiento al clima organizacional. Rev Cuba Enferm [Internet]. 2018 [citado 2020 jun 05];34(1). Disponible en: http://www.revenfermeria.sld.cu/index.php/enf/article/view/1257/336

4. Banks GC, McCauley KB, Gardner WL, Guler CE. A meta-analytic review of authentic and transformational leadership: a test for redundancy. Leadersh Q. 2016;27(4):634-52. doi: https://doi.org/10.1016/j.leaqua.2016.02.006

5. Neves TMA, Parreira PMSD, Graveto JMN, Freitas MJS, Rodrigues VJL. Nurse managers' perceptions of nurse staffing and nursing care quality: a cross-sectional study. J Nurs Manag. 2020; 28(3):625-33. doi: https://doi.org/10.1111/jonm.12966

6. Landman C, Arriola Y, Chacón A, Giorgis De A, Esparza C, Herrera E, et al. Transitando hacia el ejercicio de autonomía y liderazgo: "para el posicionamiento hay que luchar». Enferm Univ. 2019;16(2):157-70. doi: https://doi.org/10.22201/eneo.23958421e.2019.2.644

7. Robaee N, Atashzadeh-Shoorideh F, Ashktorab T, Baghestani A, Barkhordari-Sharifabad M. Perceived organizational support and moral distress among nurses. BMC Nurs [Internet]; $2018 \quad$ [cited $2020 \quad$ Apr 23$] ; 17(2): 2-7 . \quad$ Available from: https://bmcnurs.biomedcentral.com/articles/10.1186/s12912-017-0270-y

8. Iglesias Almenteros AL, Torres Esperón JM. Intervención para la mejora del clima organizacional en enfermería. Infodir [Internet]. 2019 [citado 2020 jun 11];15(28):98-108. Disponible en: https://www.medigraphic.com/pdfs/infodir/ifd-2019/ifd1928i.pdf

9. Hamui-Sutton A, Varela-Ruiz M. La técnica de grupos focales. Investigación Educ Médica [Internet]. 2013 [citado 2018 ago 22];2(5):55-60. Disponible en: http://www.scielo.org.mx/scielo.php?script=sci_arttext\&pid=S2007-50572013000100009\&lng=es 
10. Bardin L. Análise de conteúdo: a visão de Laurence Bardin. Rev Eletrônica Educ [Internet]. 2012 [acesso em 2020 jun 15];6(1):383-7. Disponível em: http://www.reveduc.ufscar.br/index.php/reveduc/article/view/291/156

11. Bardin L. Análisis de contenido. Madrid: Ediciones Akal; 2002.

12. Hernandez Ruiz A, Castillo Cuello JJ, Rodriguez Acuña M, González Hernández A. Observancia de la ética en investigaciones presentadas en jornadas científicas del hospital clínico quirúrgico Joaquín Albarrán. Rev Habanera Cienc Médi [Internet]. 2020 [citado 2020 mar 14];19(2):1-12. Disponible en: http://www.revhabanera.sld.cu/index.php/rhab/article/view/3008

13. Orgambídez A, Almeida H. Predictores del compromiso organizacional en enfermería: resultados de Portugal. Inv Educac Enferm. 2018;36(1). doi: https://doi.org/10.17533/udea.iee.v36n1e14

14. Lui JNM, Johnston JM. Validation of the nurse leadership and organizational culture (N-LOC) questionnaire. BMC Health Serv Res. 2019;19:469. doi: https://doi.org/10.1186/s12913-019-4290-z

15. Asiri SA, Rohrer WW, Al-Surimi K, Da'ar O, Ahmed A. The association of leadership styles and empowerment with nurses' organizational commitment in an acute health care setting: a cross-sectional study. BMC Nurs; 2016;15(38):2-10. doi: https://doi.org/10.1186/s12912-016-0161-7

16. Choi EA, Kim E-K, Kim PB. Effects of the educational leadership of nursing unit managers on team effectiveness: mediating effects of organizational communication. Asian Nurs Res (Korean Soc Nurs Sci). 2018;12(2):99-105. doi: https://doi.org/10.1016/j.anr.2018.03.001

17. Keogh TJ, Robinson JC, Parnell JM. Assessing behavioral styles among nurse managers: implications for leading effective teams. Hosp Top. 2019;97(1):23-38. doi: 10.1080/00185868.2018.1563460

18. Van Bogaert P, Van Heusden D, Peremans L, Verspuy M, Kureckova V, Van de Cruys Z, et al. Predictors of burnout, work engagement and nurse reported job outcomes and quality of care: a mixed method study. BMC Nurs. 2017;16(5). doi: https://doi.org/10.1186/s12912-016-0200-4

19. Salar AR, Ahmadi F, Navipour H. Turbulent atmosphere-based dominant management behavior of the head nurses in clinical wards: a qualitative study. 2016. Iran Red Crescent Med J.18(2):e21754. doi: 10.5812/ircmj.21754

20. Gómez Gómez R. Evolución y perfiles de la enfermera quirúrgica y gestora. Arch Mem [Internet]. 2019 [citado 2020 abr 03];16:e12103. Disponible en: http://ciberindex.com/index.php/am/article/view/e12103e/e12103e 
Liderazgo \& clima organizacional de un hospital pediátrico cubano. Visión de ... 22

21. Cruz Rivero C. La naturaleza del cuidado humanizado. Enfermería (Montev). 2020;9(1):2132. doi: https://doi.org/10.22235/ech.v9i1.2146

22. Román López P. La comunicación en los gestores de enfermería: un papel fundamental. Rev Esp Comun Salud [Internet]. 2011 [citado 2020 jun 09];2(1):46-54. Disponible en: https://erevistas.uc3m.es/index.php/RECS/article/view/3404/2055

23. Díaz Piñera AM, Rodriguez Salva AJ, Balcindes Acosta S, De Vos P, Van der Stuyfs P. Clima organizacional: percepción por enfermeras del nivel primario de atención. Rev Cuba Enferm [Internet]. 2016 [citado 2020 jun 12];32(3). Disponible en: http://www.revenfermeria.sld.cu/index.php/enf/article/view/967

24. Marante, LRP. A reconstrução do sentido de comunidade: implicações teórico-metodológicas no trabalho sobre a experiência de sentido de comunidade [dissertação]. Lisboa: Universidade de Lisboa; 2010 [acesso em 2020 jun 12]. Mestrado em Psicologia Comunitária. Disponível em: http://repositorio.ul.pt/bitstream/10451/2643/1/ulfp037471_tm.pdf

25. Wandebroocke ACNS, Baasch C, Antunes MC, Menezes M. O sentido de comunidade em uma equipe multiprofissional hospitalar: hierarquia, individualismo, conflito. Trab Educ Saúde. 2018;16(3):1157-76. doi: http://dx.doi.org/10.1590/1981-7746-sol00155

26. Villagra Rivera N, Ruoti Cosp M. Empoderamiento enfermero en las unidades de cuidados intensivos en el Hospital Central del Instituto de Previsión Social. Mem Inst Investig Cienc Salud. [Internet]. 2018 [citado 2020 jun 12];16(1):84-93. Disponible em: https://pdfs.semanticscholar.org/113e/1dfe949c17ab0407552bc1467d1bd69f6a4b.pdf

Editor científico jefe: Cristiane Cardoso de Paula

Editor científico: Tânia Solange Bosi de Souza Magnago

\section{Autor correspondiente}

Julia Maricela Torres Esperón

E-mail: mtorresesperon7@gmail.com

Enderezo: Calle 202.No. 5116. E./51 y 59. La Lisa, La Habana, Cuba

CEP: 10070

\section{Contribuciones de Autoria}

\section{1 - Annia Lourdes Iglesias Armenteros}

Confección y diseño del estudio/investigación, Análisis e interpretación de datos, revisión final con participación crítica e intelectual en el manuscrito. 


\section{2 - Julia Maricela Torres Esperón}

Confección y diseño del estudio / investigación, Análisis e interpretación de datos, revisión final con participación crítica e intelectual en el manuscrito.

\section{3 - Yuliett Mora Pérez}

Análisis e interpretación de datos, revisión final con participación crítica e intelectual en el manuscrito.

\section{4 - Jose Rolando Sánchez Rodríguez}

Análisis e interpretación de datos, revisión final con participación crítica e intelectual en el manuscrito.

\section{Como citar este artículo}

Armenteros LI, Esperón JMT, Pérez YM, Rodríguez JRS. Liderazgo \& clima organizacional de un hospital pediátrico cubano. Visión de enfermeras jefas y asistenciales. Rev. Enferm. UFSM. 2020 [Acesso en: Año Mes Día]; vol.10 e79: 1-23. DOI: https://doi.org/10.5902/21797692IdArtigo 